\title{
A Case report of Class II Division 2 Malocclusion in Adult women treated by Fixed appliance with CIA and removable anchor plate incorporated bite plane: Biomechanical Considerations
}

\author{
Sattar MH'1 BDS, DDS, MCPS
}

\begin{abstract}
This article describes our treatment of Class II, division 2 adult patients requiring premolar extractions. Division 2 cases are often characterized by severe deep bites, lingually inclined upper central and lower incisors, and labially flared maxillary lateral incisors. This patients also tend to exhibit problems with the upper and lower occlusal planes, such as deep curves of Spee, High lip line, marked labiomental depression. Because of the deep bite and supra eruption of the maxillary incisors, the gingival margins of the maxillary anterior teeth are malaligned, and the lingually inclined mandibular incisors have excessively high gingival margins ( Fig. 1 ).
\end{abstract}

\begin{abstract}
The treatment protocol for this patients includes extraction of premolars both upper and lower in right side to relieve crowding, with simultaneous correction of the deep bite by intrusion of the upper and/or lower incisors. Intrusion mechanics are performed with a bite opening bend on a preformed nickel titanium arch wire. Space closure is accomplished with power chain and guard behind the extracted site in anchor plate. Extraction of upper premolar and lower 1st molar (tooth no 36) in left side was done earlier. A 21 years old women with Cl-II Div-II malocclusion type B came to Dental Centre, Dhaka, with chief complaint of an unhappy smile. Retroclined 4 Incisors, Deep bite, Crowding, deficient lower facial height, Gummy smile and a moderately convex hard- and soft-tissue profile because of a retrusive mandible with over jet of $1.5 \mathrm{~mm}$ and over bite of $6 \mathrm{~mm}$ was observed. The mechanics plan should be individualized based on the specific treatment goals. Camouflage Treatment was done with the help of an anchor plate incorporated anterior incline plane. Intrusion mechanics are performed with preformed nickel titanium Connecticut Intrusion Arch (CIA) and anchor plate incorporated bite plane. Treatment was successfully completed with extractions of both pre-molars in right side and left lower 1st molar (Tooth no 36) and upper 1st premolar(Tooth no 24) already extracted ( Fig. 2 A) before starting of orthodontic treatment. Treatment of 20 months which improves incisor inclination, Deep bite correction; eliminate crowding, normal smile line and improvement of gummy smile. With the above mentioned protocol normal inclination of both upper-lower incisor, normal over jet and over bite were also achieved.
\end{abstract}

Key words: Cl-II Div-2 malocclusion, Anchor plate, Connecticut Intrusion Arch (CIA), Cl-II skeletal base, Deep bite, Crowding, Gummy smile and straight lateral profile. (Ban J Orthod \& Dentofac Orthop, April 2011; Vol-1, No. 2, 1824)

\section{INTRODUCTION AND LITERATURE REVIEW}

A Class II division 2 malocclusion is characterized by retroclination of two or more maxillary incisors, retroclination of the lower incisors, a Class II molar relationship and an increased overbite (van der Linden, 1983). One of the most important features is the high position of the lower lip in relation to the maxillary incisors ( Luffi ngham, 1982 ; van der Linden, 1983; Lapatki et al. , 2002). Orthodontic treatment of Class II division 2 malocclusions is known to be difficult and prone to relapse ( Canut and Arias, 1999 ). Maintenance of post-treatment results is reported to depend upon the intensity and direction of mandibular growth during and after retention ( Riedel, 1960 ; Payne, 1964 ; Schudy, 1968 ; Simons and Joondeph, 1973 ). Some authors have found a significant correlation between the long-term stability of treated Class II division 2 malocclusions and a relative decrease in lower lip coverage of the maxillary incisors ( Mills, 1973 ; Fletcher, 1975 ; Luffingham, 1982 ; van der Linden, 1986 ; Selwyn-Barnett, 1991 ; Lapatki et al. , 2002 ).
As relapse is often observed after retention, the height of the lip line could be one of the causative factors. However, the influence of forces exerted by the lips, cheeks, and tongue on the position of the teeth is still the subject of scientific debate (Weinstein et al. , 1963 ; Proffit, 1978 ). Stability of upper incisor inclination changes in Class II division 2 patients.

Two case reports illustrate the effective treatment of Class II division 2 malocclusion with modifications to the Twin Block appliance. This approach may reduce the total treatment time and reduce the need for extra-oral anchorage and cl-II elastics. In each of the cases presented treatment has been carried out on a non-extraction basis with full correction of the malocclusion. ${ }^{1}$ Study was to evaluate the changes in incisor inclination following orthodontic treatment in Class II division 2 patients, and to assess the long-term stability after retention. The mean change in incisor inclination during orthodontic treatment was 15.2 degrees. There was a mean relapse of 2.2 degrees between just after treatment T2 and 3.5 years (mean) post-treatment T3, but 
this was not considered clinically relevant. The amount of relapse was independent of the type of the retention appliance (Hawley type retainer or lingual retainer).

The relationship between long-term stability of the incisor inclination and between the lip line relative to the upper incisors was also investigated based on cephalometric data. A mean decrease in lip line height of $0.6 \mathrm{~mm}$ was seen at the end of treatment $\mathrm{T} 2$ and, although the observed change was statistically significant, it would not be clinically relevant. ${ }^{2}$

Treatment of Class II malocclusion in early mixed dentition has always planned on growth modification, such as functional appliances, are used to retard or redirecting maxillary growth and simultaneously stimulating mandibular growth. ${ }^{3-5}$ On the other hand, in adult patients with severe Class II malocclusions, generally involving extremely deficient mandibles, orthognathic surgery is often the only possible treatment.

Although camouflage may be attempted by extracting premolars, the soft-tissue objectives may be impossible to meet. Even so, a recent study has shown that patient satisfaction with camouflage treatment was similar to that achieved with surgical mandibular advancement. ${ }^{6}$ In Class II patients with mild-to-moderate skeletal discrepancies, dental compensation may well be the treatment of choice. Common treatment procedures for such patients include flaring of incisors, interproximal tooth reduction, and extractions.

Treatment of an adult Class II patient requires careful diagnosis and a treatment plan involving aesthetic, occlusal and functional considerations. ${ }^{7-9}$ The treatment objectives must include the chief complaint of the patient, and the mechanics plan should be individualized based on the specific treatment goals.
At our office, we have designed an anchor plate added bite plane. We also use multifunctional orthodontic wires capable of simultaneously performing different orthodontic tooth movements because both the force system and the side effects of these "smart" wires are now well understood, we can usually avoid the need for headgear and cl-II elastic.

This article describes our treatment of Class II, division 2 adult patients requiring premolar extractions. Division 2 cases are often characterized by severe deep bites, lingually inclined upper central and lower incisors, and labially flared maxillary lateral incisors in some cases. This patients also tend to exhibit problems with the upper and lower occlusal planes, such as deep curves of Spee, high lip line, marked labiomental depression and retroclined all incisors. Because of the deep bite and supra eruption of the maxillary incisors, the gingival margins of the maxillary anterior teeth are usually malaligned, and the lingually inclined mandibular incisors may have excessively high gingival margins ( Fig. 1 ).

The treatment protocol for these patients includes extraction of upper premolars in the right side both upper and lower to relieve crowding, with simultaneous correction of the deep bite by intrusion of the upper and/or lower incisors. Extraction of upper premolar and lower 1st molar (tooth no 36) in left side was done earlier. At the University of Connecticut, they have designed multifunctional orthodontic wires CIA/CNA capable of simultaneously performing different orthodontic tooth movements which can usually avoid the need for headgear and Class II elastics. Intrusion mechanics can be performed with either a bite opening bend on preformed nickel titanium normal or said Connecticut Intrusion $\mathrm{Arch}^{10}$ (CIA) or CNA beta titanium arch wires which is not avilable in our country. Space closure is accomplished with power chain and guard behind the extracted site in anchor plate and/or CNA mushroom-loop wires or CNA T-loops.

21 Year Old Women Presenting Cl-II Division 2 Malocclusion, Important Clinical Considerations And Mechanics
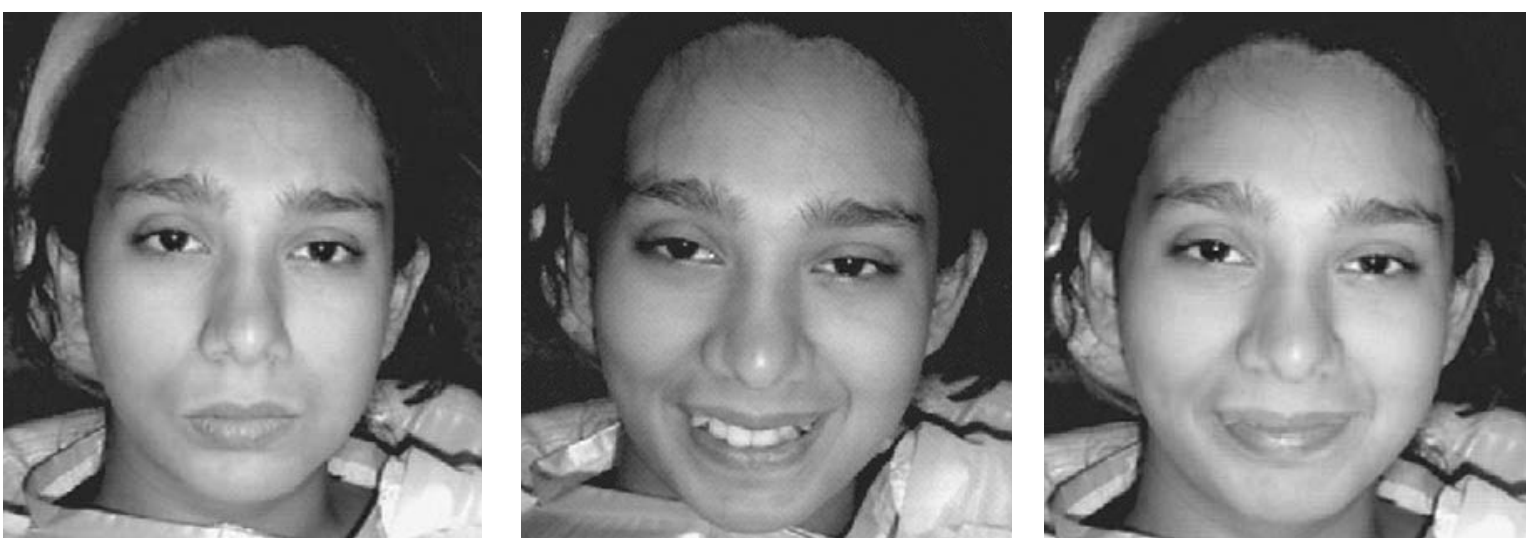

Fig. 1 A. Smile displaying un-aesthetic gingival margin heights (Extra Oral). 
A Case report of Class II Division 2 Malocclusion in Adult women treated by Fixed appliance with CIA and removable anchor plate incorporated bite plane: Biomechanical Considerations
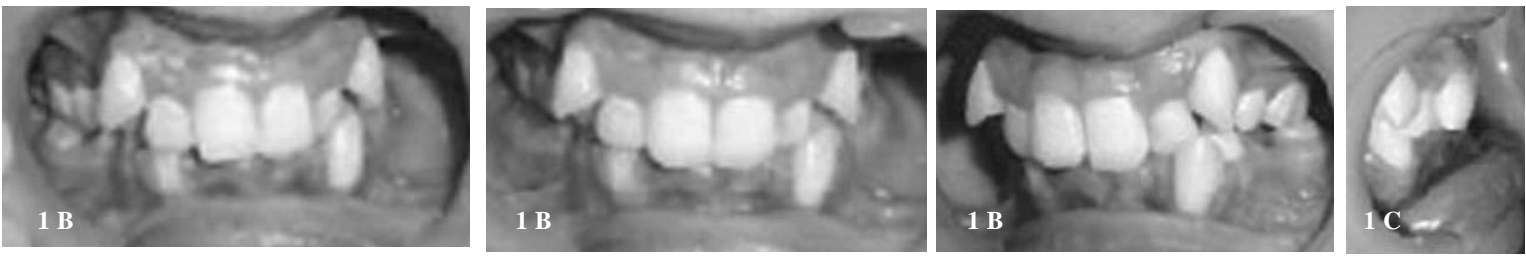

Fig. 1 B. Intra Oral photographs before treatment. , Fig. 1 C. over jet of $1.5 \mathrm{~mm}$ and over bite of $6 \mathrm{~mm}$

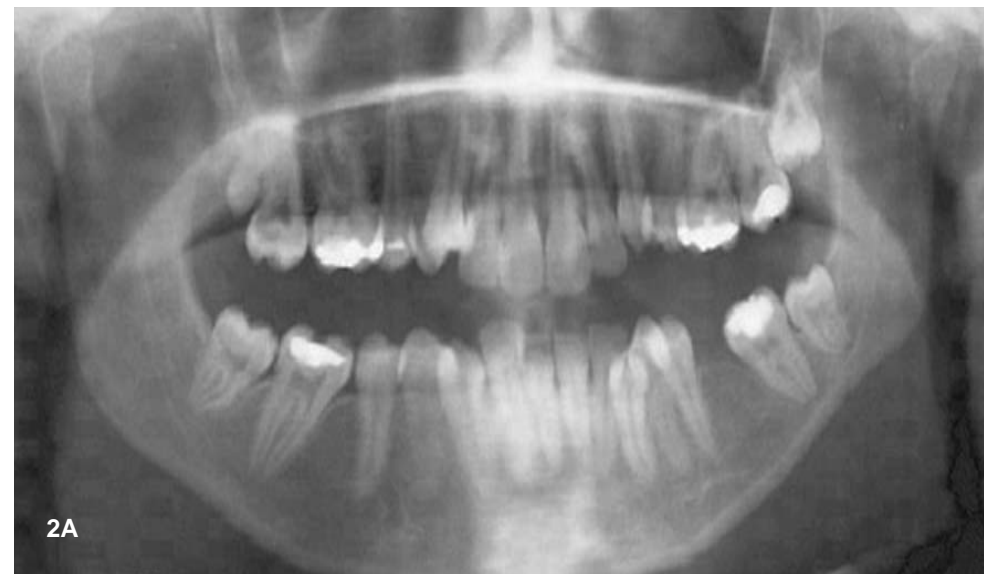

Fig. 2 A. lower 1st molar (Tooth no 36) and upper 1st premolar (Tooth no 24) left side already extracted before starting of orthodontic treatment

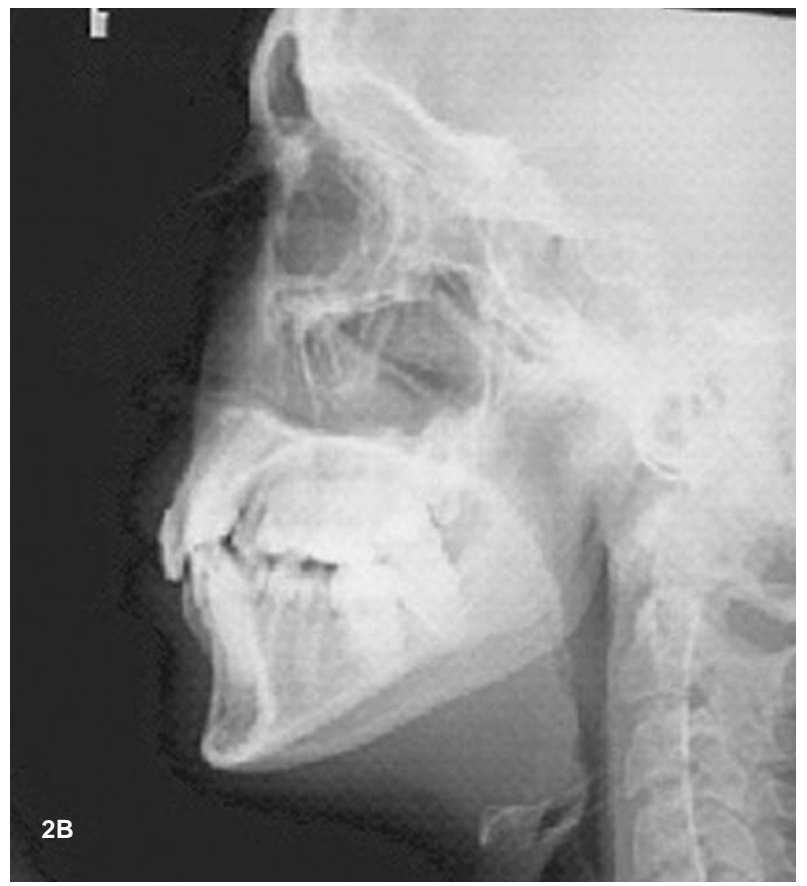

Fig. 2 B. a moderately convex hard- and soft-tissue profile because of a retrusive mandible
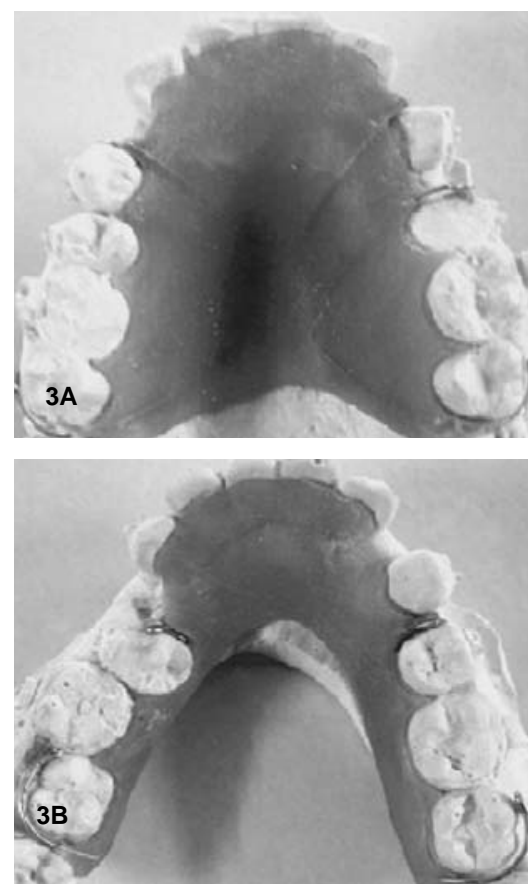

Fig. 3 A, B. Anchor plate with C clasp on 2nd molar and guard behind the extraction site. 

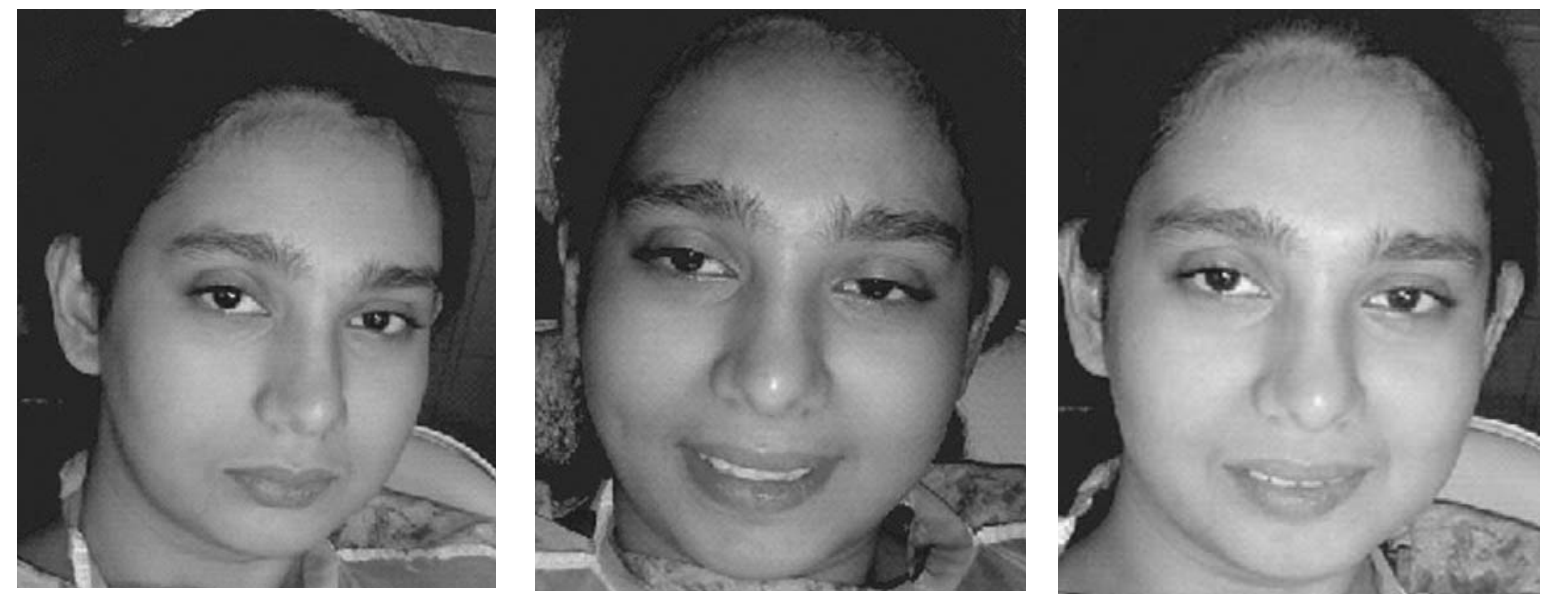

Fig. 4 Smile displaying aesthetic gingival margin heights (Extra Oral).
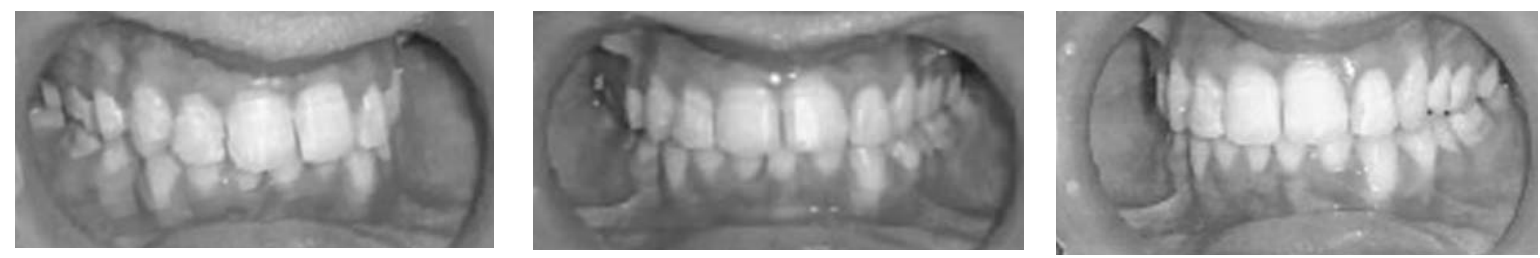

Fig. 5 Intra oral photographs after treatment

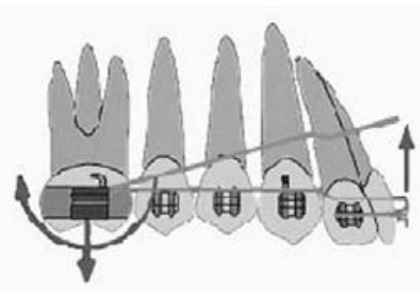

Fig. 6: Intrusion arch produces anterior tipback moment and intrusive force along with extrusive force on molars.

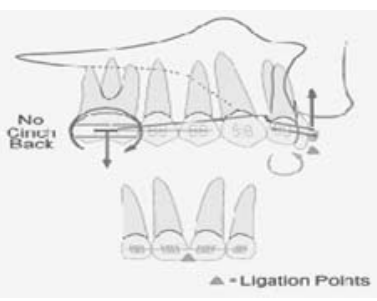

Fig. 7: Force system and ligation points of intrusion arch in Class II, division 2 malocclusion.

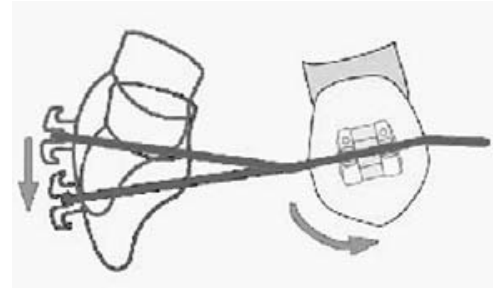

Fig. 8: Canine retraction generates extrusive effect on incisors. To counteract this tendency, intrusion arch is tied anteriorly.
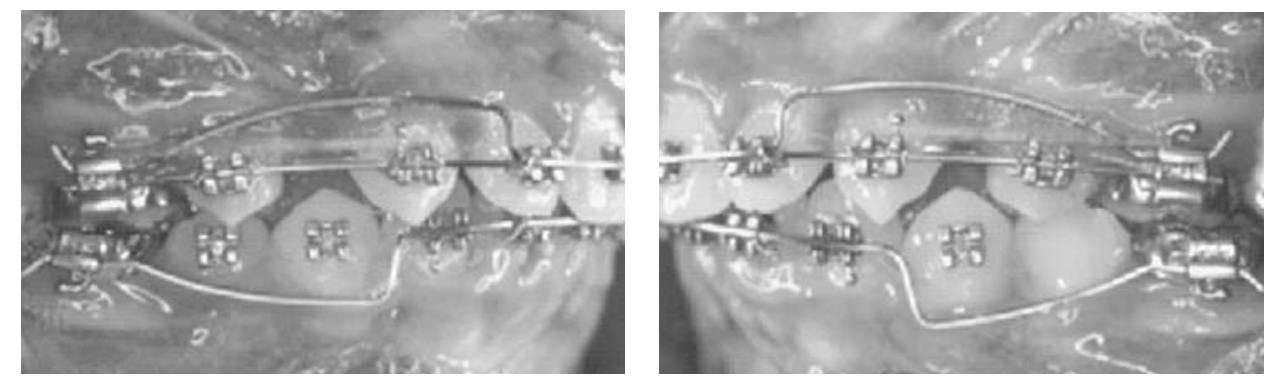

Fig. 9 A: Canine retraction with .016" × .022" stainless steel base arch and overlay intrusion arch for anchorage and incisor control. 
A Case report of Class II Division 2 Malocclusion in Adult women treated by Fixed appliance with CIA and removable anchor plate incorporated bite plane: Biomechanical Considerations
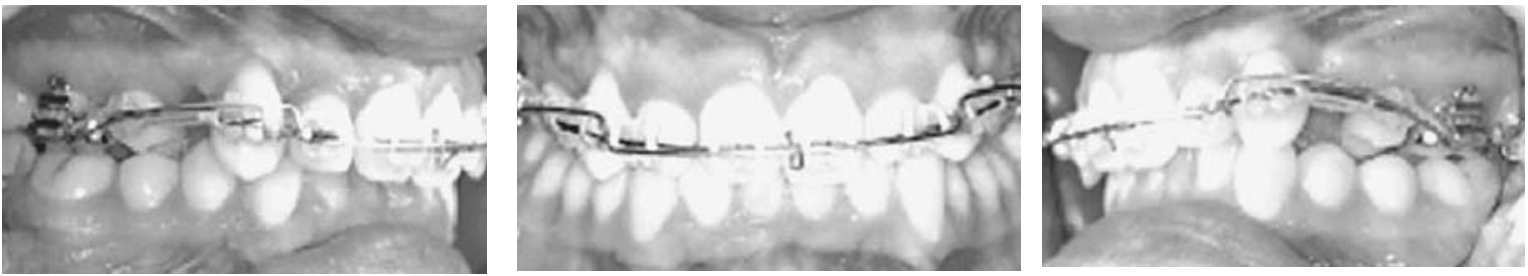

Fig 9 B: After initial intrusion phase (note incisor level and molar tipback), .016" × .022" stainless steel base arch is used with short .017" $\times .025 "$ nickel titanium intrusion arch to retract canines.
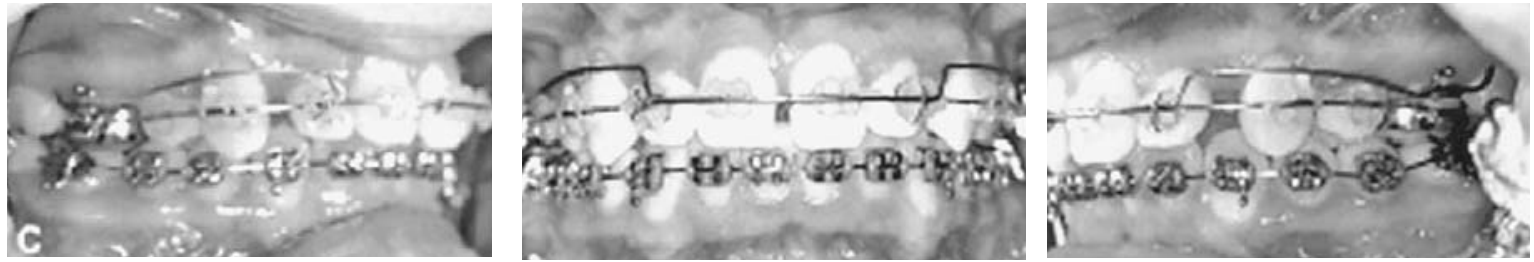

Fig. 9 C: Canines fully retracted into Class I positions. Note intrusion, overbite, and anchorage control without elastic wear.

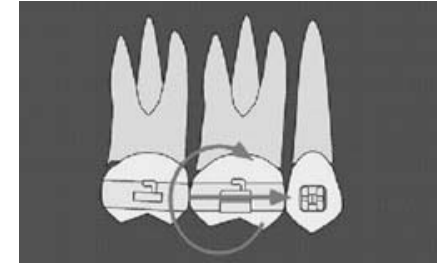

Fig.10: Moment at molar counteracts mesial reactive force in anchor unit.

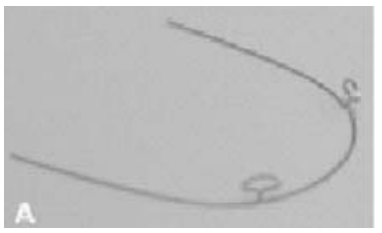

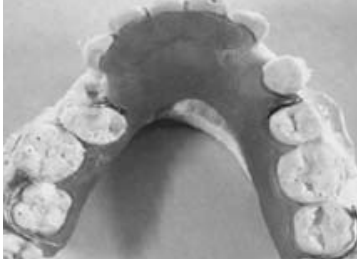

Fig.11: Anchor plate C clasp on 2nd molar and guard behind extraction site

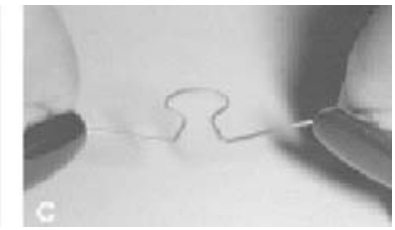

Fig. 12 A: Mushroom-loop archwire without preactivation bends. B. Archwire with gable bends mesial and distal to archwire. C. $3 \mathrm{~mm}$ preactivation of loop.
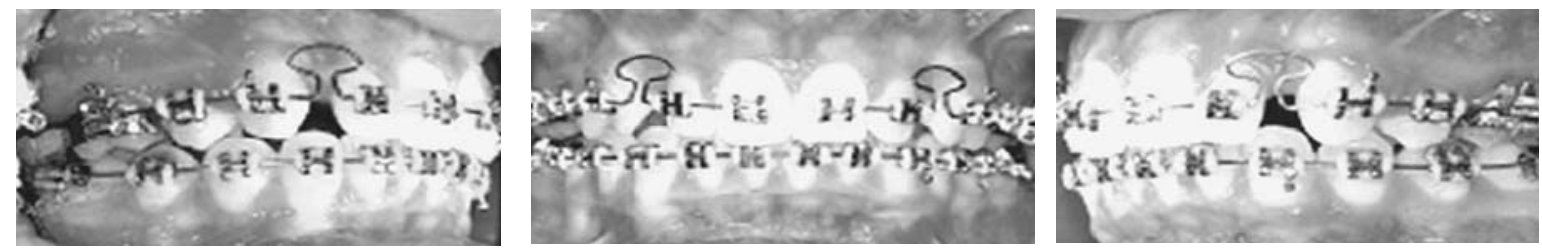

Fig. 13: Mushroom-loop archwire with spaces closed. Wire is left in place for another six weeks to allow residual moments to deliver proper axial root inclinations. B. Same patient with ideal axial inclinations.

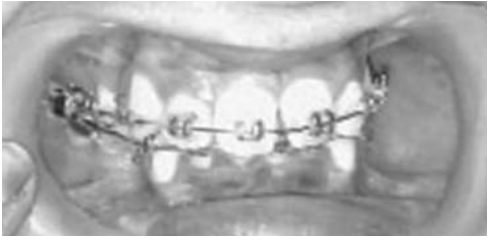

22

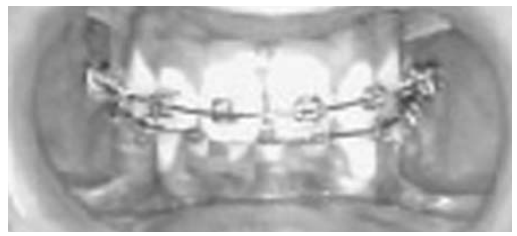

Fig.14: Arch coordination and finishing

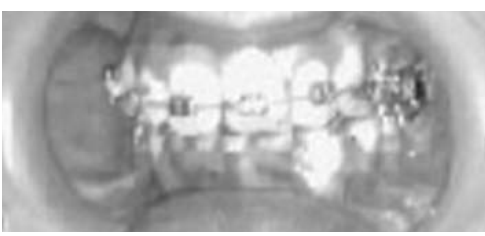

Bangladesh Journal of Orthodontics and Dentofacial Orthopedics (BJO \& DFO)

Vol. 1, No. 2, April 2011 


\section{ANCHOR PLATE AND INTRUSION ARCH WIRES CIA}

Anchor plate is an acrylic palatal/lingual plate with a C clasp on 2nd molar and guard posterior to extraction site (Fig 3 A B). Oral mucosa, facet surface of teeth, $\mathrm{C}$ clasp and guard combined to act as an unit and increase anchorage value. In every follow-up acrylic plate was trimmed to achieve desire teeth movement. Anterior bite Plane, Anterior Incline Plane, Expansion screw or any lingual clasp may also be added to have a desired movement of tooth/ teeth. Incorporated anterior incline plane will reduce (OB) over bite as well procline lower incisors.

Preformed CIA nickel titanium intrusion wires deliver a force of $35-40 \mathrm{~g}$ in patients with average arch length and a full complement of teeth. Short CIA wires are used in extraction cases where spaces have already been closed, and long wires are used in non extraction cases. The length of the wire is determined by the location of the moment bent into it. For ideal force activation, the bend should be $3-5 \mathrm{~mm}$ mesial to the first molar auxiliary tube when the wire is inserted ( Fig. 7 ). To produce higher force levels of 50-60g, which might be desirable in some adult patients, preformed CNA beta titanium arch wires can be activated by placing moment bends in front of the molar tubes. The bends can be increased or decreased to vary the magnitude of force. The anterior portion of the intrusion arch is tied to an anterior segment in the incisor brackets (usually .017"x.025" stainless steel), depending on the anterior-posterior dental objectives. One of the multifunctional aspects of intrusion wires is that they can be used for flaring the incisors when needed. Because the upper central incisors are lingually inclined in Class II, division 2 patients, the intrusion arch should not initially be bend distal to the molar tubes, so that the incisors can be flared prior to their intrusion (Fig. 8). The wire can then be bend/ cinched back 2-3mm distal to the molar tubes for intrusion of the incisors. It is also important to ligate the intrusion arch initially to the anterior segment between the two central incisors. This allows the point of attachment of the wire to be as anterior as possible in relation to the incisors' center of resistance. Once the incisor root inclinations have been corrected, the intrusion arch can be ligated to the anterior segment at the two lateral incisors and between the central incisors.

\section{CANINE RETRACTION MECHANICS}

After flaring the incisors and intrusion of the incisors, the anterior teeth can be retracted in one of two ways: en masse retraction of the six anterior teeth, or a two-step procedure involving canine retraction followed by retraction of the four incisors. In this article, only the two-step method will be described.

A stainless steel base archwire is used to slide the canines distally. To prevent the incisor bite from deepening due to the change in inclination of the canines (Fig. 9), an intrusion arch can be tied over the stainless steel wire ( Fig. 10A ). The intrusion arch is ligated at the level of the lateral incisors and between the central incisors, delivering a distal crown tipback moment on the molars to effectively control the loss of distal anchorage often associated with sliding mechanics (Fig. 11). These mechanics are ideal for cases where anchorage is critical and, in adults, can eliminate the need for headgear or Class II elastics. With an intrusive force on the incisors and a moment on the molars, the base arch wire will not deflect too much, as is often seen in sliding mechanics due to the friction generated by canine retraction.

Mushroom-Loop Space-Closing Archwires

At this stage, in the majority of patients, the four incisors either need translation or controlled differential movement of their crowns and root apices. For translatory movement, a high, constant moment-to-force ratio of approximately $10: 1$ is recommended. ${ }^{11}$ If the ratio is too low, as is common with straightwire mechanics, the incisor crowns will move lingually, reducing the overjet and giving an erroneous impression of a tooth-size discrepancy when the spaces distal to the lateral incisors appear to be enormous. To correct this side effect, either the bite must be reopened or the incisors must be torqued, creating an unnecessary stress at the root apices as well as lengthening the treatment time.

The preformed mushroom-loop space-closing archwire produces an ideal moment-to-force ratio. The mushroom shape of the loop will not interfere with the gingival tissue, and an activated loop will not become distorted, thus improving force delivery. For .022" brackets, an .017"x.025" CNA archwire comes preformed with the mushroom loops at standardized distances of $26-46 \mathrm{~mm}$ in increments of $2 \mathrm{~mm}$ ( Fig. 13A ). This measurement represents the distance between the distal surfaces of the lateral incisors across the midline.

Once the proper archwire has been selected, it is preactivated outside the mouth to achieve the proper moment-to-force ratio. First, the legs of both mushroom loops are carefully separated by about $3 \mathrm{~mm}$. Additional gable bends may be placed mesially as needed to increase the anterior moment (torque), and distal to the mushroom loop to increase the anchorage moment ( Fig. 13B ). Next, the torque on the distal legs is eliminated. The archwire is then placed in the mouth and engaged across the arch from first molar to first molar (Fig. 14 ). Another $1 \mathrm{~mm}$ of activation is added, for a total of $4 \mathrm{~mm}$.

The loop should not be reactivated until at least $3 \mathrm{~mm}$ of space has been closed, thus maintaining a more constant moment-toforce ratio. After the spaces are completely closed, the wire should be left in the mouth for one or two additional visits, so that the residual moments can be used to correct the axial root inclinations of the anterior and posterior teeth. This completely eliminates the need for root uprighting and torquing springs and significantly shortens treatment time.

\section{FINISHING AND RETENTION}

The finishing phase of treatment simply involves the use of coordinated .017"x.025" or .018"x.025" NiTi wires. Minor bends can be placed in these titanium wires for detailing the alignment and occlusion. The finishing stage is usually short because of the correct positioning of the incisors after retraction ( Fig. 5 ).

In adult patients, a maxillary modified Hawley wraparound 
retainer is ideal, because there is no interference in the occlusion. A lower bonded 3-3 retainer is recommended. It is important to emphasize that intrusion is a stable movement ${ }^{12}$; minor overbite relapse should be expected, however, since its correction does involve some posterior buccal extrusion.

\section{CASE REPORT}

A 21-year-old female presented with the chief complaint of "unhappy smile" (Fig. 1). She had a moderately convex hard- and soft-tissue profile because of a retrusive mandible. A Class II, division 2 malocclusion was associated with a decrease over jet and $100 \%$ deep bite due to moderately supra-erupted upper incisors and excessively supra-erupted lower incisors. The upper incisors were upright, and the lower incisors normally inclined. Both arches exhibited mild-to-moderate crowding.

The treatment objective in this case was to maintain the hard- and soft-tissue profiles. In the vertical dimension, the goal was to intrude the maxillary incisors to improve the lip-to-incisor relationship and achieve a flat occlusal plane. The lower incisors needed to be intruded slightly, but extrusion of the posterior buccal segments was undesirable. In the anteroposterior dimension, the treatment objectives were to maintain the upper incisor crown positions and move the roots lingually. The lower incisors required minor intrusion as well as flaring. The molar positions, arch width, and midlines needed to be maintained.

The upper first premolars were extracted to relieve crowding. An .017"x.025" nickel titanium intrusion arch was placed to simultaneously flare and intrude the upper incisors. Group A anchorage $^{13}$ (critical) was maintained with the intrusion arch during cuspid retraction, using sliding mechanics on an .016"x.022" stainless steel archwire (Fig. 10B,C). The mushroom loops in an .017"x.025" NiTi archwire were preactivated as described above (Fig. 14). Posterior moments in the mushroom-loop archwires helped maintain anchorage during upper incisor retraction. The mandibular crowding was resolved by aligning the lower arch. Finishing was accomplished in two visits with coordinated upper and lower .017"x.025" NiTi archwires (Fig. 4,5).

\section{CONCLUSION}

Treatment of Class II, division 2 malocclusion in adults is always challenging. Relapse is quite common if we ignore incisor inclination and inter incisal angle. Applying proper knowledge of biomechanical principles to execute the mechanics plan/protocol is the key to achieve predictable results with minimal side effects. The appliance shown in this article like anchor plate, CIA can be applied in a variety of situations with only minor alterations. By using the biomechanical concepts presented here an anchor plate and a set of arch wires designed with specific objectives in mind, the clinician can achieve the desired goals.

\section{RECOMMENDATIONS}

Use of Anchor bite plane and CIA is new to us. Taking in mind the cultural and social values and affordability of this south east Asian region we propose a simpler appliance like anchor plate. Anchor plate appliance and CIA archwire will reduce the need of TPA (Transpalatal arch), head gear and sometimes Cl-II elastic. Further research is recommended to proof the efficacy, efficiency and affectivity of this new appliance.

\section{REFERENCES}

1. F. M.V. Dyer et al. The Modified Twin Block Appliance in the Treatment of Class II Division 2 Malocclusions JO Vol 28 No. 4 p 271-280, 2001 UK Email: dyer.sheffield@virgin.net

2. H . Devreese et al. Stability of upper incisor inclination changes in Class II division 2 patients, European Journal of Orthodontics 29 (2007) 314-320, 2007 E-mail: guy.depauw@UGent.be

3. Staley, R.: Etiology and prevalence of malocclusion, in Textbook of Orthodontics, ed. S. Bishara, W.B. Saunders Co., Philadelphia, 2001, p. 83

4. Baccetti, T.; Franchi, L.; McNamara, J.A.; and Tollaro, I.: Early dentofacial features of Class II malocclusion: A longitudinal study from the deciduous through the mixed dentition, Am. J. Orthod. 111:502-509, 1997.

5. McNamara, J.A.: Components of Class II malocclusion in children 8-10 years of age, Angle Orthod. 51:177-202, 1981.

6. Mihalik, C.A.; Proffit, W.R.; and Phillips, C.: Long-term follow-up of Class II adults treated with orthodontic camouflage: A comparison with orthognathic surgery outcomes, Am. J. Orthod. 123:266278, 2003.

7. Nanda, R.: Biomechanics and Esthetic Strategies in Clinical Orthodontics, ed. R. Nanda, in press,W.B. Saunders Co., Philadelphia.

8. Kuhlberg, A. and Glynn, E.: Treatment planning considerations for adult patients, Dent. Clin. N. Am. 41:17-28, 1997.

9. Nanda, R.: Correction of deep overbite in adults, Dent. Clin. N. Am. 41:67-88, 1997.

10. Nanda, R.; Marzban, R.; and Kuhlberg, A.: The Connecticut Intrusion Arch, J. Clin. Orthod. 32:708-715, 1998.

11. Smith, R.J. and Burstone, C.J.: Mechanics of tooth movement, Am. J. Orthod. 85:294-307, 1984.

12. Burzin, J. and Nanda, R.: The stability of deep overbite correction, in Retention and Stability in Orthodontics, ed. R. Nanda and C.J. Burstone, W.B. Saunders, Philadelphia, 1993, p. 61.

13. Nanda, R.: Biomechanics in Clinical Orthodontics, ed. R. Nanda, W.B. Saunders Co., Philadelphia, 1997.

\section{Correspondence}

Dr. Mostaque Hassan Sattar BDS, DDS, MCPS

Associate Professor

Department of Orthodontics \& Dentofacial Orthopedics,

Dhaka Dental College \& Hospital.

E-mail : mhsattar2000@gmail.com 